\title{
REARRANGING MEASURES
}

\section{G. BROWN ${ }^{1}$ and J. H. WILLIAMSON}

(Received 16 September 1981)

Communicated by W. Moran

\begin{abstract}
A churning transformation can be defined on probability measures by an infinite sequence of finite permutations of mass. Continuity and absolute continuity of measures are invariants for such transformations but it is shown that certain probability measures whose Fourier-Stieltjes transforms fail to vanish at infinity may be churned into measures whose transforms do vanish in this sense.
\end{abstract}

1980 Mathematics subject classification (Amer. Math. Soc.): 28 A 65, 42 A 72, 60 E 05.

\section{Introduction}

Suppose that $\mu$ is a probability measure on the unit interval $[0,1[$. Consider the partition of $\left[0,1\left[\right.\right.$ into $n$ equal intervals, $I_{1}=\left[0,1 / n\left[, I_{2}=\left[1 / n, 2 / n\left[, \ldots, I_{n}=\right.\right.\right.\right.$ $[(n-1) / n, 1[$. In a great number of practical situations one is concerned with the set of numbers $\left\{\mu\left(I_{k}\right)\right\}$ rather than with the sequence $\left(\mu\left(I_{k}\right)\right)_{k=1}^{n}$. It follows that the object of interest is often not so much $\mu$ itself but rather those measures which can be derived from $\mu$ by certain rearrangements of mass. Here we shall make that statement precise and obtain some basic properties of the allowable rearrangements. It turns out that continuity and absolute continuity are invariants but that the property of having a Fourier transform which vanishes at infinity fails to be invariant even for the simplest subclass of rearrangements.

\footnotetext{
'The research of the first-named author was supported by the Science Research Council (grant No. A87791)

C. Copyright Australian Mathematical Society 1983
} 


\section{Background and definitions}

Let us start with the simplest possible case which we shall call churning. Taking the measure $\mu$ on $\left[0,1\left[\right.\right.$, we divide the support into two equal intervals ( $\left[0, \frac{1}{2}[\right.$ and $\left[\frac{1}{2}, 1[)\right.$ and write $\mu$ as the sum, $\mu_{1}+\mu_{2}$, of the corresponding restrictions. Now we have a choice. Either we leave things as they are or we interchange $\mu_{1}$ and $\mu_{2}$ (more precisely we replace $\mu$ by the measure $\delta_{1 / 2} * \mu_{1}+\delta_{1 / 2} * \mu_{2}$ ). Having chosen, we proceed to the next stage where the two intervals are both bisected and in each case we may either leave the mass distribution unaltered or interchange the left hand restriction measure with the right hand restriction measure. The process is continued for an infinite number of steps and a limit measure obtained.

For modelling purposes we should use a wider class of rearrangements. At the $n$th stage of an inductive process we will have described a partition of [0, 1[ into $k_{n}$ intervals labelled from the left as $I_{n}(k), k=0, \ldots, k_{n}-1$, and a permutation $\Pi(n)$ on $\left\{0, \ldots, k_{n}-1\right\}$. The rearranged measure $\nu$ will be characterized by

$$
\nu\left(I_{n}(k)\right)=\mu\left(I_{n}\left(\Pi(n)^{-1}(k)\right)\right), \quad k=0, \ldots, k_{n}-1 ; n=1, \ldots
$$

In fact at the first stage we choose a positive integer $k_{1}$ and divide [0, 1[ into $k_{1}$ congruent intervals. We also choose a permutation $\Pi(1)$ of $\left\{0, \ldots, k_{1}-1\right\}$. At stage $n+1$ we take each interval $I_{n}(k)$, choose a positive integer $j(n, k)$ and divide $I_{n}(k)$ into $j(n, k)$ congruent intervals $J(n, k ; j), j=0, \ldots, j(n, k)-1$, which we permute according to some permutation $\Pi(n, k)$ of $\{0, \ldots, j(n, k)-1\}$. Now let

$$
k_{n+1}=\sum_{k=1}^{k_{n}} j(n, k) .
$$

We have obtained $k_{n+1}$ intervals forming a partition of [0, 1[ and a permutation of these sending $J(n, k ; j)$ to $J(n, \Pi(n)(k) ; \Pi(n, \Pi(n)(k))(j))$. Now we relabel the intervals $J(n, k ; j)$ (for varying $k$ and $j$ ) from the left as $I_{n+1}(k)$ (for $\left.k=0, \ldots, k_{n+1}-1\right)$ and we label the induced permutation of $\left\{0, \ldots, k_{n+1}-1\right\}$ by $\Pi(n+1)$. This completes the inductive step.

Before discussing some simple properties of rearrangements we consider two typical examples which motivate the definition. (Related notions are discussed in [4].) In the first case let us take the distribution of ore in a mineral deposit. Although the geographical disposition of the ore is important and much studied, the most commonly available tabulated information is the frequency distribution of ore grades. It is this passage from a "spatial" probability distribution to a frequency distribution which corresponds to the rearrangement process. 
To see this let us suppose we have a squared grid covering the area of the deposit and a function which assigns to each square the total quantity of ore below that square. [In practice we may regard this quantity as being estimated by a sample drilling whose cross-section is a small disc centred at the centre of the square.] By normalizing we obtain a probability measure on a finite $\sigma$-algebra (the grid) and by passing to the limit over successive refinements we obtain a measure $\mu$, say, on a measurable space which can be identified with the Borel space $[0,1[$. [In practice the drill cross-section sets a bound to attainable refinements.] For any particular set of values urior a grid we suppose that the ore densities are divided into bands and the frequency distribution of these bands is tabulated. In principle this frequency distribution is derived from (the Radon-Nikodym derivative with respect to area measure of) the restriction of $\mu$ to the grid. Let us now envisage a sequence of investigations where we have full knowledge of spatial results but where an outside observer tries to guess $\mu$ from the frequency distribution tables. He is obliged to choose some permutation of the values of $\mu$ to the first grid. With our knowledge of the spatial configuration of the higher densities we will drill with greater frequency in these areas, so in the second drilling some are refined further than others. When presented with the results our outside observer makes a new guess consistent with his first and this is what corresponds to permutations within the squares of the previous stage. [In general the numerical data would disclose how the values at stage two should be clumped to correspond to stage one but this could be disguised by accidental Diophantine equations. We take no account of such serendipity.] The process continues to the next stage and so on. It should now be clear how this matches our definition of rearrangement.

The second example concerns income. Here we use a finite generalized decimal . $x_{1} x_{2} \ldots x_{n}$ to encode characteristics of income earners. The first digit let us say takes the value 0 for male and 1 for female, the second digit takes a value in say $\{0,1,2, \ldots, 8\}$ to represent a particular age band and so on. Again we pass to an idealized limit and envisage normalized income as a probability measure $\mu$ on $\left[0,1\left[\right.\right.$ whose points are given infinite expansions.$x_{1} x_{2} \ldots x_{n} \ldots$ In principle we consider the partition of $[0,1$ [ correspond to "decimals" of length $N$ and use the associated restriction of $\mu$ to determine a frequency distribution for income. This distribution would also arise from a rearrangement of $\mu$.

It was shown in [4] that relatively mild constraints on $\mu$ in the above examples ensure that the resulting frequency distributions are asymptotically lognormal as refinements increase. The present discussion indicates that these constraints need only apply to some rearrangement of $\mu$.

We close this section by showing that generalized decimal expansions of a sort can be used in any rearrangement and that the rearrangement is implemented by a map of the underlying interval. In particular this shows that each rearrangement 
does lead to a measure! As a preliminary convenience we restrict the definition of rearrangement by demanding that all the integers $k_{n}, j(k, n)$ are strictly greater than one. This simply means that we really do refine at each stage and each substage. From a modelling point of view this may seem not entirely desirable; for example we might choose to take no more sample drillings in a particularly unpromising region. However the only cases where this matters correspond to intervals which remain undisturbed from some stage onwards, and we achieve the same end result by continuing to divide but insisting on using the trivial (identity) permutation at each stage. In other words we have not restricted the class of rearrangements after all.

Proposition 1. Let $\mu$ be a (regular Borel) probability measure on [0, 1[. Every rearrangement of $\mu$ is of the form $\theta^{*}(\mu)$, where $\theta:[0,1] \rightarrow[0,1[$ is measurable and

$$
\theta^{*}(\mu)(E)=\mu\left(\theta^{-1}(E)\right) \quad(\text { E Borel }) .
$$

Proof. The generalized decimal expansions which we consider will correspond to the types of nested partition discussed in our definition of rearrangement. To see what is required let us set the question of permutations on one side and consider an arbitrary point $x$ of $[0,1$. At stage $1, x$ belongs to some interval, say, $I_{1}\left(x_{1}\right)$ where $x_{1} \in\left\{0, \ldots, k_{1}-1\right\}$. At stage $2, x$ belongs to, say, $J\left(1, x_{1} ; x_{2}\right)$ which has another label of the form $I_{2}(k)$. Let us write $s\left(x_{1}, x_{2}\right)$ for that particular $k$. At stage $n+1, x$ belongs to $J\left(n, s\left(x_{1}, x_{2}, \ldots, x_{n}\right) ; x_{n+1}\right)$ which is also labelled $I_{n+1}\left(s\left(x_{1}, x_{2}, \ldots, x_{n+1}\right)\right)$. Since $x$ belongs to a unique interval at each stage this process defines the sequences $\left(x_{n}\right),\left(s\left(x_{1}, \ldots, x_{n}\right)\right)$. We may say that $x$ has the expansion.$x_{1} x_{2} \ldots x_{n} \ldots$ In the special case where $x_{n}=0$ for all $n \geqslant N+1$, say, we may as well say that $x$ has the finite expansion.$x_{1} x_{2} \ldots x_{N}$. Note that, in this case, $x$ is the left hand end-point of the interval $I_{n}\left(s\left(x_{1}, \ldots, x_{N}\right)\right)$. Thus $s\left(x_{1}, \ldots, x_{n}\right)$ counts the number of end-points of intervals in the first $n$ stages which lie to the left of the end-point which has the finite expansion.$x_{1} x_{2} \ldots x_{n}$.

Notation so far has anticipated a reasonable interpretation as generalized decimals. To achieve this we require a numerical description of the branching process which determines a typical nest of partitions. In fact we should prescribe a sequence $\left(t_{n}\right)$ of maps taking values in the positive integers greater than or equal to two. $t_{1}$ is constant and the domain of $t_{2}$ is $\left\{0,1, \ldots, t_{1}-1\right\}$. For $n \geqslant 1$, $\left(x_{1}, \ldots, x_{n+1}\right)$ belongs to the domain of $t_{n+2}$ if and only if $\left(x_{1}, \ldots, x_{n}\right)$ belongs to the domain of $t_{n+1}$ and $x_{n+1} \in\left\{0, \ldots, t_{n+1}\left(x_{1}, \ldots, x_{n}\right)-1\right\}$. Given such a family $\left(t_{n}\right)$ we admit as a generalized decimal any expression.$x_{1} x_{2} \ldots x_{n} \ldots$ in which $x_{n+1} \in\left\{0, \ldots, t_{n+1}\left(x_{1}, \ldots, x_{n}\right)-1\right\}$ and $x_{n+1} \neq t_{n+1}\left(x_{1}, \ldots, x_{n}\right)-1$ infinitely often. There is a one-to-one correspondence between such generalized decimals and the points of $[0,1[$. 
Let us check the last assertion. Given $\left(t_{n}\right)$, we first define $s\left(x_{1}, \ldots, x_{n}\right)$ which assigns to $\left(x_{1}, \ldots, x_{n}\right)$ its lexicographical order position in the domain of $t_{n+1}$. Then we obtain all the partitions in our earlier construction by setting $k_{1}=t_{1}$ and $j\left(n, s\left(x_{1}, \ldots, x_{n}\right)\right)=t_{n+1}\left(x_{1}, \ldots, x_{n}\right)$. It is clear from our earlier discussion that each $x$ in [0, 1[ gives rise to an allowable expansion. $\left(x_{n+1}=t_{n+1}\left(x_{1}, \ldots, x_{n}\right)-1\right.$ eventually, is impossible since the corresponding intervals have empty intersection.) Conversely, given an allowable expansion.$x_{1} x_{2} \ldots x_{n} \ldots$, we take the intervals $I_{n}\left(s\left(x_{1}, \ldots, x_{n}\right)\right)$, observe that we may add the right hand end-points without affecting the intersection, and apply Cantor's lemma to see that there is a unique point $x$ which belongs to them all.

Now we turn to the problem of describing the map which implements a rearrangement. Since the rearrangement changes the branch process under consideration we will describe how to map a point $x$ with expansion.$x_{1} x_{2} \ldots x_{n} \ldots$ relative to a partition described by $\left(t_{n}\right), s$, etcetera to a point $y=\theta(x)$, whose expansion is $y_{1} y_{2} \ldots y_{n} \ldots$ relative to $\left(t_{n}^{\prime}\right), s^{\prime}$ etcetera. The prime refers to the new partition produced by the rearrangment. Since the interval $I_{n}(k)$ is replaced by the interval $I_{n}(\Pi(n)(k))$, we find that

$$
t_{n+1}^{\prime}\left(y_{1}, \ldots, y_{n}\right)=j\left(n, \Pi(n)^{-1} s^{\prime}\left(y_{1}, \ldots, y_{n}\right)\right) .
$$

((2:3) refers to an arbitrary point $y$ with expansion.$\left.y_{1} y_{2} \ldots y_{n} \ldots\right)$ To define $\theta$, we specify that

$$
y_{1}=\Pi(1)\left(x_{1}\right), \quad s^{\prime}\left(y_{1}, \ldots, y_{n}\right)=\Pi(n)\left(s\left(x_{1}, \ldots, x_{n}\right)\right) .
$$

Since $s^{\prime}$ has been defined inductively by (2.3), it follows that, given $\left(x_{n}\right),(2.4)$ defines $\left(y_{n}\right)$ uniquely. It is easy to see that $\theta$ maps the finite expansion.$x_{1} \ldots x_{n}$ to the finite expansion.$y_{1} \ldots y_{n}$, and the interval $I_{n}\left(s\left(x_{1}, \ldots, x_{n}\right)\right)$ onto the interval $I_{n}\left(s^{\prime}\left(y_{1}, \ldots, y_{n}\right)\right)$. In view of (2.4) this last statement translates as

$$
\theta\left(I_{n}(k)\right)=I_{n}(\Pi(n)(k)), \quad k=0, \ldots, k_{n}-1 .
$$

Now that $\theta$ is seen to be a measurable bijection of $[0,1[$, it is clear that $\theta$ induces a map $\theta^{*}$ of measures. Comparing (2.1) and (2.5) we see that $\theta^{*}(\mu)$ is the required rearrangement of $\mu$.

\section{Invariants}

Now that we have seen that each rearrangement of $\mu$ leads to another measure, it is appropriate to ask which properties of a measure remain invariant under rearrangement. 
Proposition 2. Let $\mu$ be a probability measure on $\left[0,1\left[\right.\right.$ and let $\nu=\theta^{*}(\mu)$ be a rearrangement of $\mu$. Then

(i) $\mu$ is continuous if and only if $\nu$ is continuous

(ii) $\mu$ is discrete if and only if $\nu$ is discrete

(iii) $\mu$ is absolutely continuous with respect to Lebesgue measure

if and only if $\nu$ is absolutely continuous with respect to Lebesgue measure. In fact if $\mu$ has Radon-Nikodym derivative $f$ with respect to Lebesgue measure, then $\nu$ has derivative $f \circ \theta$.

RFMARK. By way of counterpoint, it should be noted that there exist (singular) measures $\mu$ whose support is all of $[0,1]$, and a rearrangement $\theta^{*}(\mu)$ such that $\theta^{*} \mu \perp \mu$. (This can occur even in the case of churning and will be justified in the next section.)

Proof. Let us write,

$$
\mu=\mu_{d}+\mu_{c},
$$

where $\mu_{d}$ is the discrete component, $\mu_{c}$ the continuous component of $\mu$. It is straightforward to see that $\theta^{*}$ is additive. In particular

$$
\boldsymbol{\theta}^{*} \boldsymbol{\mu}=\boldsymbol{\theta}^{*} \mu_{d}+\boldsymbol{\theta}^{*} \mu_{c} \text {. }
$$

Moreover, for the probability atom $\delta_{x}$ concentrated at $x$, we have

$$
\theta^{*} \delta_{x}=\delta_{\theta(x)} .
$$

These remarks show that $\theta^{*} \mu_{d}$ is a discrete measure, which maps to $\mu_{d}$ under $\left(\theta^{-1}\right)^{*}$. Since $\theta^{*} \mu_{c}$ maps to $\mu_{c}$ under $\left(\theta^{-1}\right)^{*}$, we also see that $\theta^{*} \mu_{c}$ can have no discrete component. In other words

$$
\theta^{*} \mu_{d}=\left(\theta^{*} \mu\right)_{d}, \quad \theta^{*} \mu_{c}=\left(\theta^{*} \mu\right)_{c} .
$$

We have proved (i) and (ii).

The truth of (iii) follows from the fact that Lebesgue measure $\lambda$ on $[0,1[$ is invariant under rearrangement. Suppose in fact that $\mu$ is absolutely continuous with respect to $\lambda$ and that $f$ is the derivative. Then

$$
\frac{d \theta_{\mu}^{*}}{d \lambda}=\frac{d \theta_{\mu}^{*}}{d \theta_{\lambda}^{*}}=f \circ \theta \text {. }
$$

REMARK. It is possible to formulate the previous result in terms of $L$-homomorphism. An $L$-homomorphism $\Theta: M(\mathbf{R}) \rightarrow M(\mathbf{R})$ (where $M(\mathbf{R})$ denotes the regular bounded Borel measures on $\mathbf{R}$ ) is a positive linear map which is norm-preserving on positive measures and has the property that, given $0 \leqslant \omega \leqslant \theta \mu$, there exists $\nu \geqslant 0$ such that $\theta \nu=\omega . \theta^{*}$ is an $L$-homomorphism under which $\lambda$ is invariant and which preserves the $L$-spaces $m_{c}(\mathbf{R}), M_{d}(\mathbf{R}), L^{1}(\mathbf{R})$. We shall see that $\theta^{*}$ does not 
preserve the $L$-space $M_{0}(\mathbf{R})$ of measures whose transforms vanish at infinity. (A closed linear subspace $N$ of $M(\mathbf{R})$ is an $L$-space if $\mu \in N, \nu \ll \mu$ implies $\nu \in N$.)

\section{Churning and coin tossing}

With the main object of discussing the effect of rearrangment of the Fourier transform we now focus attention on a special class of rearrangements, those coming from churning; and a special class of measures, those coming from coin-tossing. [Since the object is to exhibit properties which are not preserved we should specialize as much as possible.]

The infinite convolution

$$
\mu=\underset{n=1}{*}\left[p_{n} \delta_{-2^{-n}}+q_{n} \delta_{2^{-n}}\right]
$$

where $p_{n}+q_{n}=1, p_{n}, q_{n}>0$, can be thought of as distributing mass over $\left[-1,1\left[\right.\right.$ in the following way. At the first stage total mass $p_{1}$ is assigned to the left hand interval [ $-1,0\left[\right.$ and mass $q_{1}$ is assigned to the right hand interval [0, 1[. At the next stage the mass $p_{1}$ is distributed between the sub-intervals $\left[-1,-\frac{1}{2}[\right.$, [ $-\frac{1}{2}, 0$ [ in the proportion $p_{2}$ to $q_{2}$ and the mass $q_{1}$ is redistributed left to right between $\left[0, \frac{1}{2}\left[\right.\right.$ and $\left[\frac{1}{2}, 1\left[\right.\right.$ in the same proportion $p_{2}$ to $q_{2}$. Thus the four intervals at stage two have been assigned masses $p_{1} p_{2}, p_{1} q_{2}, q_{1} p_{2}, q_{1} q_{2}$ reading from the left. By continuing in this way we eventually obtain the measure $\mu$. [We may make the link with coin-tossing by thinking of $\mu$ as the distribution of a numerical random variable whose binary digits have been determined by independent tosses of a sequence of biassed coins.] It is clear that churning will take us outside the class of measures described by (4.1). For example at stage two we might have $p_{1} q_{2}$, $p_{1} p_{2}, q_{1} p_{2}, q_{1} q_{2}$ and this cannot arise from a convolution. Churning itself is more special than binary rearrangements, for example, $p_{1} p_{2}, q_{1} p_{2}, p_{1} q_{2}, q_{1} q_{2}$ cannot be achieved by churning. (It is assumed that we start from the first two terms in the product defined in (4.1).)

The measure corresponding to that given by (4.1) but with $p_{n}, q_{n}$ interchanged for all $n$ is obtained by churning. In fact the new measure is of the form $\theta^{*} \mu$ for $\theta(x)=-x$. In the case that

$$
\sum\left(\frac{1}{2}-p_{n}\right)^{2}=\infty,
$$

it is easy to see, using standard results for sums of independent random variables, that $\mu$ is singular to Lebesgue measure and that $\theta^{*} \mu$ is singular to $\mu$.

The main result of [1] is that $\mu$ vanishes at infinity if and only if $\frac{1}{2}-p_{n}$ tends to zero. A simpler argument appears as a remark in [2] and the result is generalized by the first part of Theorem 2 of [3]. 
It is necessary to establish some notation for further work. We shall be concerned with the class of all measures obtained by churning from a given measure of coin-tossing type. To this end we fix a sequence $\left(\alpha_{n}\right)_{n=1}^{\infty}$ of real numbers such that $0<\alpha_{n}<1$. For each positive integer $n$, we let

$$
W_{n}=\left\{\sum_{j=1}^{n} \varepsilon_{j} 2^{-j}: \varepsilon_{j}= \pm 1\right\} \text {. }
$$

Each measure in the class will correspond to a sequence $\left(\phi_{n}\right)_{n=0}^{\infty}$ of maps $\phi_{n}: W_{n} \rightarrow\{-1,1\}$. [Take $W_{0}=\{0\}$.] In fact let us write, for $\omega \in W_{n}$,

$$
\sigma_{n}(\omega)=\frac{1}{2}\left(1+\phi_{n}(\omega) \alpha_{n+1}\right) \delta_{-2^{-n-1}}+\frac{1}{2}\left(1-\phi_{n}(\omega) \alpha_{n+1}\right) \delta_{2^{-n-1}} .
$$

Define

$$
\mu_{1}=\sigma_{0}(0)=\frac{1}{2}\left(1+\phi_{0}(0) \alpha_{1}\right) \delta_{-1 / 2}+\frac{1}{2}\left(1-\phi_{0}(0) \alpha_{1}\right) \delta_{1 / 2},
$$

and if $\mu_{n}=\Sigma_{\omega \in W_{n}} m_{n}(\omega) \delta_{\omega}$ define

$$
\mu_{n+1}=\sum_{\omega \in W_{n}} m_{n}(\omega)\left(\delta_{\omega} * \sigma_{n}(\omega)\right)=\sum_{\omega \in W_{n+1}} m_{n+1}(\omega) \delta_{\omega},
$$

and

$$
\mu=\lim _{n \rightarrow \infty} \mu_{n} \text {. }
$$

[By taking $p_{n}=\frac{1}{2}\left(1+\alpha_{n}\right), \phi_{n}(\omega) \equiv 1$, we obtain the measure $\mu$ of (4.1).]

LEMMA 1.

$$
m_{n}(\omega)=\prod_{k=0}^{n-1} \frac{1}{2}\left(1-\phi_{k}\left(\omega^{(k)}\right) \varepsilon_{k+1} \alpha_{k+1}\right)
$$

where

$$
\omega^{(k)}=\sum_{j=1}^{k} \varepsilon_{j} 2^{-j}, \quad \omega^{(0)}=0 .
$$

In the special case when $\phi_{n} \equiv 1$, we find that

$$
|\hat{\mu}(\theta)|^{2} \leqslant\left|\hat{\mu_{n}}(\theta)\right|^{2} \leqslant \prod_{j=1}^{k}\left\{\cos ^{2}\left(\frac{\pi \theta}{2^{n-j}}\right)+\alpha_{n+1-j}^{2} \sin ^{2}\left(\frac{\pi \theta}{2^{n-j}}\right)\right\},
$$

for all $k<n$. (Here $\rho \hat{(}(\theta)=\int \exp (2 \pi i \theta x) d \rho(x)$.)

Let us restrict attention to measures for which it is possible to derive an inequality

$$
\left|\mu \hat{\wedge}\left(x 2^{n}\right)\right| \leqslant C \prod_{j=1}^{k} F\left(2^{j} x, \alpha_{n-j}\right), \quad \text { all } k<n, \text { when }|x| \leqslant 2 .
$$


Such a measure will be called admissible if the following technical condition is satisfied: if $(q(n))$ is an increasing sequence of positive integers, $\varepsilon$ is strictly positive, and, for all positive integers $N$,

$$
\lim \sup _{n} \prod_{j=1}^{N} F\left(2^{j} x, \alpha_{q(n)-j}\right) \geqslant \varepsilon,
$$

then $2^{j} x$ tends to 0 modulo one as $j \rightarrow \infty$.

LEMMA 2. Let $F(\theta, \alpha)^{2}=\max \left\{\cos ^{2}(2 \pi \theta)+\alpha^{2} \sin ^{2}(2 \pi \theta), \sin ^{2}(2 \pi \theta)+\right.$ $\left.\alpha^{2} \cos ^{2}(2 \pi \theta)\right\}$, and suppose that $\lim \sup \alpha_{n}<1$. Then $\mu$ is admissible.

Lemma 3. Let $F(\theta, \alpha)=\max \{|\cos 2 \pi \theta|+\alpha|\sin 2 \pi \theta|,|\sin 2 \pi \theta|+\alpha|\cos 2 \pi \theta|\}$ and suppose that $\alpha_{n} \rightarrow 0$ for $n$ lying outside a fixed set $X=\left\{x_{k}\right\}$ such that $x_{k+1}-x_{k} \rightarrow \infty$ as $k \rightarrow \infty$. Then $\mu$ is admissible.

LEMma 4. Suppose that $\mu$ is admissible and that $\hat{\mu}$ does not vanish at infinity. Then there exist an odd integer $K$, a sequence of real numbers $\left(k_{n}\right)$, and a sequence of positive integers $(p(n))$ such that $\left|\mu\left(k_{n} 2^{p(n)}\right)\right|$ is bounded away from zero and $k_{n} \rightarrow K$.

Proof. Suppose that $\mu$ does not tend to zero at infinity. Then there is some $\varepsilon>0$ and a sequence $\left(y_{n}\right)$ of real numbers, such that $\left|y_{n}\right| \rightarrow \infty$ and

$$
\left|\mu \hat{(}\left(y_{n}\right)\right| \geqslant \varepsilon, \quad n=1,2, \ldots
$$

Since we might replace $K$ by $-K$, there is no essential loss of generality in supposing that $y_{1}>2, y_{n} \rightarrow+\infty$. With this done, let us write

$$
y_{n}=x_{n} 2^{q(n)}
$$

where $1 \leqslant x_{n}<2$, and $q_{n}$ is a positive integer. Some subsequence of $\left(x_{n}\right)$ converges to a number $x$ in [1,2]. Let us pass to this subsequence.

For (large) $N$ and $q(n)>N$, we see that

$$
\varepsilon \leqslant \prod_{r=1}^{N} F\left(x_{n} 2^{r}, \alpha_{q(n)-r}\right) .
$$

Letting $n \rightarrow \infty$ in the last inequality we find

$$
\varepsilon \leqslant \lim \sup \prod_{r=1}^{N} F\left(x 2^{r}, \alpha_{q(n)-r}\right) .
$$

At this stage we free $N$ to see that

$$
\cos \left(2 \pi \times 2^{r}\right) \rightarrow 1 \quad \text { as } r \rightarrow \infty
$$


and hence that $x$ is a dyadic rational of the form $x=K 2^{-l}$, where $K$ is an odd integer, $l$ is a nonnegative integer. Let us now define $k_{n}=2^{\prime} x_{n}, p(n)=q(n)-l$ (restarting the sequence at some suitable $n_{1}>1$ if necessary) to obtain the stated result.

Proposition 3. If $\alpha_{n} \rightarrow 0$ then $\mu \in M_{0}$.

Proof. Let $(p(n)),\left(k_{n}\right)$, and $K$ be as in Lemma 4. In view of that lemma there exists some positive $\varepsilon$ such that

$$
\left|\mu^{\wedge}\left(k_{n} 2^{p(n)}\right)\right|^{2} \geqslant 2 \varepsilon^{2}, \quad n=1,2, \ldots
$$

and, for each $n$, an integer $m(n)$ which may be chosen greater than $p(n)$, such that

$$
\left|\hat{\mu_{m(n)}}\left(k_{n} 2^{p(n)}\right)\right|^{2} \geqslant \varepsilon^{2}, \quad n=1,2, \ldots
$$

while

$$
K\left|\alpha_{j}\right|<\varepsilon, \quad j \geqslant p(n) .
$$

Now use the fact that

$$
\left|\hat{\mu_{n+k}}(y)\right| \leqslant\left|\hat{\mu_{n+k-1}}(y)\right|+\alpha_{n+k}|y| 2^{-n-k}
$$

to estimate $\hat{\mu_{m(n)}}\left(K 2^{p(n)}\right)$ in terms of $\hat{\mu_{p(n)+1}}\left(K 2^{p(n)}\right)$.

\section{Fourier transforms}

In this section we consider a specific way of assigning a measure $\mu$ to a sequence $\left(\alpha_{n}\right)$. It will turn out that the Fourier-Stieltjes transform of $\mu$ vanishes at infinity provided $\alpha_{n}$ tends to zero on the complement of a set which satisfies a weak condition of lacunarity. Since the canonical coin-tossing distribution associated with $\left(\alpha_{n}\right)$ belongs to $M_{0}$ only if $\left(\alpha_{n}\right)$ is a null sequence this will show that membership of $M_{0}$ is not stable under churning.

Let us fix the choice of $\mu$ from now on. The most direct prescription of $\mu$ is as the vague limit of the measures $\mu_{n}$ given by

$$
\begin{gathered}
\mu_{n}=\sum_{\omega \in W_{n}} m_{n}(\omega) \delta_{\omega}, \\
m_{n}(\omega)=\prod_{k=0}^{n-1} \frac{1}{2}\left(1-\alpha_{k+1} \varepsilon_{k+1} \varepsilon_{k} \ldots \varepsilon_{1}\right),
\end{gathered}
$$


where the typical element of $W_{n}$ is of the form $\omega=\sum_{k=1}^{n} \varepsilon_{k} 2^{-k}$, with $\varepsilon_{k}= \pm 1$. It is a simple exercise to check that (5.1) and (5.2) are equivalent to the following condition

$$
\phi_{0}(1)=1, \quad \phi_{n+1}(\omega)=\phi_{n}\left(\omega^{(n)}\right) \varepsilon_{n+1},
$$

in which we have used the notation of the last section. Noting that (5.2) implies (5.3) corresponds to verifying that $\mu$ belongs to the class of measures obtained by churning from the canonical coin-tossing measure for which $m_{n}(\omega)$ equals $\prod_{k=0}^{n-1} \frac{1}{2}\left(1-\alpha_{k+1} \varepsilon_{k+1}\right)$.

It is also possible to describe the progressive construction of $\mu$ and the manner in which it differs from classical "Cantor-like" constructions. In fact each sub-interval at the $k$ th stage may be "lucky" (being apportioned the fraction $\frac{1}{2}\left(1+\boldsymbol{\alpha}_{k}\right)$ of the available mass) or "unlucky"(receiving the residual $\left.\frac{1}{2}\left(1-\alpha_{k}\right)\right)$. Lucky intervals are subdivided in two so that the left hand sub-interval of the next stage is unlucky and the right hand sub-interval at that stage is lucky. The corresponding statement with "lucky" and "unlucky" interchanged is also true. In a coin-tossing measure, throughout any one stage one hand is always unlucky (whether this is the right hand or the left hand may vary from stage to stage).

We require some calculations concerning the transform of $\mu$ and will make free use of the notation of the previous section.

Lemma 5. Suppose that (5.3) holds and let $\nu_{n}$ be the measure $\phi_{n} \cdot \mu_{n}$ (whose Radon-Nikodym derivative with respect to $\mu_{n}$ is $\left.\phi_{n}\right)$. Then

(i) $\hat{\mu_{n+1}}(\theta)=\cos \left(2^{-n} \pi \theta\right) \hat{\mu_{n}}(\theta)-i \alpha_{n+1} \sin \left(2^{-n} \pi \theta\right) \hat{\nu_{n}}(\theta)$,

(ii) $\hat{\nu_{n+1}}(\theta)=\sin \left(2^{-n} \pi \theta\right) i v_{n}(\theta)-\alpha_{n+1} \cos \left(2^{-n} \pi \theta\right) \hat{\mu_{n}}(\theta)$.

ProOF.

$$
\begin{aligned}
\hat{\mu_{n+1}}(\theta)= & \sum_{\omega \in W_{n}} m_{n}(\omega)\left(\delta_{\omega} * \sigma_{n}(\omega) \hat{)}(\theta)\right. \\
= & \sum_{\omega \in W_{n}^{\prime}} m_{n}(\omega) \exp (2 \pi i \theta \omega) \cos \left(2^{-n} \pi \theta\right) \\
& -i \sum_{\omega \in W_{n}} m_{n}(\omega) \phi_{n}(\omega) \exp (2 \pi i \theta \omega) \alpha_{n+1} \sin \left(2^{-n} \pi \theta\right) \\
= & \cos \left(\pi \theta 2^{-n}\right) \hat{\mu_{n}}(\theta)-i \alpha_{n+1} \sin \left(2^{-n} \pi \theta\right) \hat{\nu_{n}}(\theta) . \\
\nu_{n+1}^{\cdot}= & \sum_{\omega \in W_{n+1}} m_{n+1}(\omega) \phi_{n+1}(\omega) \delta_{\omega} \\
= & \sum_{\omega \in W_{n+1}} m_{n}\left(\omega^{(n)}\right) \frac{1}{2}\left(1-\phi_{n}\left(\omega^{(n)}\right) \varepsilon_{n=1} \alpha_{n+1}\right) \phi_{n+1}(\omega) \delta_{\omega} .
\end{aligned}
$$


Therefore we can apply (5.3) to find

$$
\begin{aligned}
\nu_{n+1}= & \sum_{\omega \in W_{n+1}} \frac{1}{2} m_{n}\left(\omega^{(n)}\right)\left(-\alpha_{n+1}\right) \delta_{\omega} \\
& +\sum_{\omega \in W_{n+1}} \frac{1}{2} m_{n}\left(\omega^{(n)}\right) \phi_{n}\left(\omega^{(n)}\right) \varepsilon_{n+1}(\omega) \delta_{\omega} .
\end{aligned}
$$

The first term in the previous sum equals

$$
\begin{gathered}
-\frac{1}{2} \alpha_{n+1}\left\{\delta_{-2^{-n-1}} * \sum_{\omega \in W_{n}} m_{n}(\omega) \delta_{\omega}+\delta_{2^{-n-1}} * \sum_{d \omega \in W_{n}} m_{n}(\omega) \delta_{\omega}\right\} \\
=-\frac{1}{2}\left(\delta_{2^{-n-1}}+\delta_{2^{-n-1}}\right) * \boldsymbol{\alpha}_{n+1} \mu_{n} .
\end{gathered}
$$

The other term in the sum for $\nu_{n+1}$ can be simplified as

$$
\frac{1}{2}\left(\delta_{2^{-n-1}}-\delta_{-2^{-n-1}}\right) * \nu_{n} .
$$

It follows that

$$
\hat{\nu_{n+1}}(\theta)=-\cos \left(2^{-n} \pi_{\theta}\right) \alpha_{n+1} \hat{\mu_{n}}(\theta)+i \sin \left(2^{-n} \pi \theta\right) \hat{\nu_{n}}(\theta) .
$$

LEMMA 6. Suppose that (5.3) holds and that $\theta_{n}=2^{n} u_{n}$, where $u_{n} \in[-u, u]$ for some $u>0$ and $u_{n}$ tends to an odd integer $K$ as $n$ tends to infinity. Suppose that $\eta>0$. Then there is a positive integer $N(\eta)$ such that, for all $n \geqslant N(\eta)$,

(i) $\max \left(\left|\hat{\mu_{m+j}}\left(\theta_{n}\right)\right|,\left|\hat{\nu_{n+j}}\left(\theta_{n}\right)\right|\right) \leqslant\left(\eta+\alpha_{n+1}\right) C, j=2, \ldots$;

(ii) $\left|\hat{\mu}_{n+j}\left(\theta_{n}\right)\right| \leqslant \eta+2 \pi u \max \left\{\left|\alpha_{n+u}\right|: u=2, \ldots, j\right\}, j \geqslant 2$;

(iii) $\left|\hat{\mu_{n+j+s}}\left(\theta_{n}\right)\right| \leqslant 2 \pi u 2^{-j}+\left|\hat{\mu_{n+j}}\left(\theta_{n}\right)\right|, j, s=1,2, \ldots$

Proof. Applying Lemma 5, we see that

$$
\hat{\mu_{n+2}}\left(\theta_{n}\right)=\cos \left(\pi / 2 u_{n}\right) \hat{\mu_{n+1}}\left(\theta_{n}\right)-i \alpha_{n+1} \sin \left(\pi / 2 u_{n}\right) \hat{u_{n+1}}\left(\theta_{n}\right)
$$

and hence that

$$
\left|\hat{\mu_{n+2}}\left(\theta_{n}\right)\right| \leqslant\left|\cos \left(\pi / 2 u_{n}\right)\right|+\alpha_{n+2}\left|\hat{\nu_{n+2}}\left(\theta_{n}\right)\right| .
$$

However

$$
\hat{\nu_{n+1}}\left(\theta_{n}\right)=-\cos \left(\pi u_{n}\right) \alpha_{n+1} \hat{\mu_{n}}\left(\theta_{n}\right)+i \sin \left(\pi u_{n}\right) \hat{v_{n}}\left(\theta_{n}\right)
$$

so

$$
\left|\nu_{n+1}\left(\theta_{n}\right)\right| \leqslant\left|\sin \pi u_{n}\right|+\alpha_{n+1} .
$$

Let us choose $N(\eta)$ such that $\left|\cos \left(\pi / 2 u_{n}\right)\right|$ and $\left|\sin \left(\pi u_{n}\right)\right|$ are less than $\frac{1}{2} \eta$, then, for $n \geqslant N(\eta)$,

$$
\left|\hat{\mu_{n+2}}\left(\theta_{n}\right)\right|<\frac{1}{2} \eta+\alpha_{n+2}\left(\alpha_{n+1}+\frac{1}{2} \eta\right) \leqslant \alpha_{n+1} \alpha_{n+2}+\eta .
$$


Also

$$
\hat{\nu_{n+2}}\left(\theta_{n}\right)=-\cos \left(\pi / 2 u_{n}\right) \alpha_{n+2} \hat{\mu_{n+1}}\left(\theta_{n}\right)+i \sin \left(\pi / 2 u_{n}\right) \hat{\nu_{n+1}}\left(\theta_{n}\right)
$$

so that

$$
\begin{aligned}
\left|\hat{\nu}_{n+2}\left(\theta_{n}\right)\right| & \leqslant\left|\cos \left(\pi / 2 u_{n}\right)\right|+\left|\hat{\nu_{n+1}}\left(\theta_{n}\right)\right| \\
& \leqslant\left|\cos \pi / 2 u_{n}\right|+\left|\sin \pi u_{n}\right|+\alpha_{n+1}
\end{aligned}
$$

where the last step used (5.4).

This gives, for $n \geqslant N(\eta)$,

$$
\left|\hat{\nu_{n+2}}\left(\theta_{n}\right)\right| \leqslant \eta+\alpha_{n+1} .
$$

Observe that (5.5) and (5.6) establish the special case of (i) corresponding to $j=2$. The triangle inequality applied to the formulae of Lemma 5 gives

$$
\begin{aligned}
& \left|\hat{\mu_{m+1}}\left(\theta_{n}\right)\right| \leqslant \max \left(\left|\hat{\mu_{m}}\left(\theta_{n}\right)\right|,\left|\hat{\nu_{m}}\left(\theta_{n}\right)\right|\right)\left(\left|\cos \theta_{n} 2^{-m} \pi\right|+\left|\sin \theta_{n} 2^{-m} \pi\right|\right), \\
& \left|\hat{\nu_{m+1}}\left(\theta_{n}\right)\right| \leqslant \max \left(\left|\hat{\mu_{m}}\left(\theta_{n}\right)\right|,\left|\hat{\nu_{m}}\left(\theta_{n}\right)\right|\right)\left(\left|\cos \theta_{n} 2^{-m} \pi\right|+\left|\sin \theta_{n} 2^{-m} \pi\right|\right),
\end{aligned}
$$

and the general case of (i) now yields to a simple inductive argument.

We turn to (ii) and use the previous lemma once more to see that, for $j \geqslant 3$,

$$
\begin{aligned}
\left|\hat{\mu_{n+j}}\left(\theta_{n}\right)\right| & \leqslant\left|\hat{\mu_{n+j-1}}\left(\theta_{n}\right)\right|+\alpha_{n+j}\left|\sin 2 \pi u_{n} 2^{-j}\right| \\
& \leqslant\left|\hat{\mu_{n+j-1}}\left(\theta_{n}\right)\right|+\alpha_{n+j} 2 \pi u 2^{-j} .
\end{aligned}
$$

If $j \geqslant 4$ this does not exceed

$$
\left|\hat{\mu}_{m+j-2}\left(\theta_{n}\right)\right|+\alpha_{n+j} 2 \pi u 2^{-j}+\alpha_{n+j-1} 2 \pi u 2^{1-j}
$$

and, in any case, for $j \geqslant 2$

$$
\begin{aligned}
\left|\hat{\mu_{n+j}}\left(\theta_{n}\right)\right| & \leqslant\left|\hat{\mu_{n+2}}\left(\theta_{n}\right)\right|+2 \pi u \max \left\{\alpha_{n+u}: u=3, \ldots\right\} \\
& \leqslant \eta+2 \pi u \max \left\{\alpha_{n+u}: u=3, \ldots\right\},
\end{aligned}
$$

provided $n \geqslant N(\eta)$. The last step is valid because we used (5.5).

To prove (iii) we note that

$$
\left|\hat{\mu_{n+j+s}}\left(\theta_{n}\right)\right| \leqslant\left|\left(\mu_{n+j+s-1}\right) \hat{n}\left(\theta_{n}\right)\right|+\left|\sin 2 \pi u_{n} 2^{-j-s}\right|
$$

and iteration gives

$$
\begin{aligned}
\left|\hat{\mu_{n+j+s}}\left(\theta_{n}\right)\right| & \leqslant\left|\hat{\mu_{n+j}}\left(\theta_{n}\right)\right|+2 \pi u\left[2^{-j-s}+\cdots+2^{-j-1}\right] \\
& \leqslant\left|\hat{\mu_{n+j}}\left(\theta_{n}\right)\right|+2 \pi u 2^{-j} .
\end{aligned}
$$


THeOREM. Let $X=\left\{x_{n}: n=1,2, \ldots\right\}$ be a subset of $\mathbf{N}$ such that $x_{n+1}-x_{n} \rightarrow \infty$ as $n \rightarrow \infty$. Suppose that $\alpha_{n} \rightarrow 0$ as $n \rightarrow \infty$ on $\mathbf{N} \backslash X$ and that $\lim \sup \alpha_{n}<1$. If $\mu$ is associated with $\left(\alpha_{n}\right)$ according to (5.1), (5.2) then $\mu \hat{~ v a n i s h e s ~ a t ~ i n f i n i t y . ~}$

Proof. We argue by contradiction, so assume $\mu$ does not vanish at infinity. In view of Lemma 4 there exists some $\varepsilon>0$, an odd integer $K$, a sequence $\left(k_{n}\right)$ converging to $K$ and a sequence $(p(n))$ of positive integers such that

$$
\left|\mu^{\wedge}\left(k_{n} 2^{p(n)}\right)\right| \geqslant 4 \varepsilon C \text {. }
$$

We shall apply Lemma 6 with $p(n)$ in place of $n$ and $u_{p(n)}=k_{n}$. In particular we fix a positive number $u$ such that $\left|k_{n}\right|$ is bounded by $u$, and we take $\eta=\varepsilon$. Now we choose $N$ such that the conclusions of Lemma 6 hold for $\theta_{p(N)}\left(=k_{N} 2^{p(N)}\right)$, and such that

$$
x, x^{\prime} \geqslant p(N) ; \quad x, x^{\prime} \in X \Rightarrow\left|x-x^{\prime}\right|>1+\log _{2}(\pi u / \varepsilon)
$$

and

$$
\boldsymbol{\alpha}_{j}<\varepsilon / 2 \pi u, \quad \text { for all } j \geqslant p(N), j \in \mathbf{N} \backslash X .
$$

If $p(N)+1$ does not belong to $X$, then, for $j=2, \ldots$,

$$
C^{-1}\left|\hat{\mu_{p(N)+j}}\left(k_{N} 2^{p(N)}\right)\right|<\eta+\alpha_{p(N)+1}<2 \varepsilon,
$$

by (i) of Lemma 6. Of course (5.10) contradicts (5.7). It remains to consider the case where $p(N)+1$ does belong to $X$. By our choice of $N$ (as specified in (5.8)) we have that $P(N)+2, p(N)+3, \ldots, p(N)+j$ belong to the complement of $X$ for some $j$ with $2 \pi u 2^{-j}$ less than $\varepsilon$. According to (ii) of Lemma 6, and (5.9)

$$
\left|\hat{\mu_{p(N)+j}}\left(k_{N} 2^{p(N)}\right)\right|<\varepsilon+(2 \pi u)[\varepsilon / 2 \pi u]=2 \varepsilon,
$$

and by (iii) of Lemma 6

$$
\left|\hat{\mu_{p(N)+j+s}}\left(k_{N} 2^{p(N)}\right)\right|<3 \varepsilon, \quad s=1,2, \ldots .
$$

For large $s$, we obtain a contradiction to (5.7), and this completes the proof.

The previous result shows that membership of $M_{0}$ is not stable under churning. Choose, for example, $\alpha_{n} \equiv \frac{1}{2}$ on $X$. Then the canonical coin-tossing measure associated with $\left(\alpha_{n}\right)$ does not belong to $M_{0}$. (Simply estimate the transform on the sequence $\left(2^{n}\right)$.) Nevertheless we are able to churn that measure to obtain a member of $M_{0}$, provided $\alpha_{n} \rightarrow 0$ on $\mathbf{N} \backslash X$. 


\section{References}

[1] J. R. Blum and B. Epstein, 'On the Fourier transforms of an interesting class of measures', Israel J. Math, 10 (1971), 302-305.

[2] G. Brown, ' $\boldsymbol{M}_{0}(G)$ has a symmetric maximal ideal off the Silov boundary', Proc. London Math. Soc. (3) 27 (1973), 484-504.

[3] G. Brown and W. Moran, 'Coin-tossing and powers of singular measures', Math. Proc. Cambridge Philos. Soc. 77 (1975), 349-364.

[4] G. Brown and J. W. Sanders, 'Lognormal genesis', J. Appl. Probability 18 (1981).

School of Mathematics

University of New South Wales

Kensington, N.S.W. 2033

Australia
Mathematics Department

Heriot-Watt University

Riccarton, Currie

Edinburgh EH1 1HX

Scotland 